\title{
Introduction: Characterization of Nonconductive Materials
}

This special issue of Microscopy and Microanalysis contains 17 articles from a topical conference on microbeam characterization of nonconductive materials. The conference was held at the Department of Mining, Metals, and Materials Engineering of McGill University in Montreal on August 2-3, 2002, as the pre-meeting congress prior to the Microscopy \& Microanalysis 2002 meeting in Quebec City, Canada.

Electrical charging is important in both imaging and analysis of polymeric, ceramic, and biological materials. It is particularly important for the photoresist materials used in the microelectronics industry. Charging affects SEM imaging of these materials because incident electrons do not flow in insulating materials. Charge accumulation induces an electrical field outside of the specimen that can deflect incident and emitted electrons making imaging difficult, if not impossible. The classical solution to this problem is to cover the specimen with a conductive coating ( $\mathrm{C}$ or Au-Pd alloy). This ground coating provides a zero potential at the surface and eliminates the field outside the material that would deflect secondary electrons. However, there is still an electrical field inside the specimen affecting secondary electron emission, which in turn can alter SEM image contrast. In microanalysis, specimen charging can alter the electron landing energy, which introduces errors in composition measurement. Even worse, these effects are often time dependent. Thus, charging cannot be ignored when analyzing nonconductive materials under an electron beam.

The microbeam analysis techniques covered here include X-ray microanalysis in the electron microprobe, low-voltage scanning electron microscopy, environmental scanning electron microscopy, and transmission electron microscopy. Many of these articles are related to the environmental scanning electron microscope (ESEM) or the variablepressure scanning electron microscope (VP-SEM). Since these microscopes allow imaging and analysis without deposition of a conductive coating, they play a leading role in the characterization of specimens susceptible to charging. In addition, a new imaging mode has been discovered with these microscopes: charge contrast imaging, which is a major theme in several of these articles. Modeling of the charging process in materials is another major topic. The time evolution of implanted and created charge is difficult to predict because of the complexity of this phenomenon; however, progress is being made, as shown in many of these articles.

I thank the 25 presenters and the 80 registered participants for making this conference a success. Appreciation also goes to the institutional supporters of the conference: McGill University, the Microscopy Society of America, the Microbeam Analysis Society, the Microscopical Society of Canada, and the European Microbeam Analysis Society.

Raynald Gauvin

Department of Mining, Metals and Materials Engineering McGill University

Montreal, Quebec, Canada 